\title{
The Nanolathe - a Dedicated Two-axis Positioner for Concentric Sample Rotation.
}

\author{
A. J. Smith ${ }^{1}$, Klaus Schock ${ }^{1}$, Stephan Kleindiek ${ }^{1}$, and Stephan Gerstl ${ }^{2}$ \\ 1. Kleindiek Nanotechnik, Reutlingen, Germany \\ 2. ScopeM, ETH Zurich, Switzerland
}

The need to make precision pillar geometries with sub-mm to micrometer dimensions is increasing due to growing fields of nanomechanical testing and increasing number of high resolution X-ray tomography facilities. The ultimate goal in continuously rotational based milling may be creating nanowires of novel materials that cannot be grown in that shape, and sharp tips with precisely defined shank angles, such as those used in Atom Probe Tomography.

Nanomechanical compression tests are often performed on pillars with non-parallel sides because they were created with a top-down annular mill. The resulting shape with varying width in depth negatively impacts the fidelity of cylindrical assumptions made for understanding the mechanical characteristics of the material. Cylinder shaped samples for high resolution X-ray tomography (e.g. [1]) application would benefit in speed of sample creation (and more symmetric cylinders may help push the techniques' resolution further). The sharp needle sample geometries required for Atom Probe Tomography (APT) are usually performed using annular milling patterns in a focused ion beam (FIB) with sequentially decreasing inner radii. This method however does not always result in high-yielding tip shapes on certain materials or material combinations, due to local changes in ion milling rates. Also, in some cases, the resulting shank angle is not ideal for the subsequent sample analysis or reconstruction (fig. 1). In order to address these issues concerning multiple fields, a novel, ultra-precise rotation module is introduced.

The difficulty in designing such a goniometric rotation device is the tool must provide the facility to position a sample's region of interest precisely on the axis of rotation. Thus the motions' stacking order must be (from bottom to top): rotation followed by $\mathrm{X}$ and $\mathrm{Y}$ translation. In standard positioner design, this either requires some manner of electrical connection to the XY translation stage positioned on top of the rotation drive that allows for unlimited $360^{\circ}$ rotation (i.e. sliding contacts or the like) or a limit on the rotation to e.g. $\pm 180^{\circ}$ due to the leads connecting to the XY translation stage.

In order to overcome this limitation a novel design was implemented in which the sample's XY translation is achieved with a linear drive that is actuated from below the rotation drive. Thus, no wiring is required on top of the rotation axis. The drive mechanism causes the sample to shift on a linear axis in two directions.

The unit is positioned inside a FIB/SEM microscope such, that the rotation drive is parallel to the electron beam (i.e. in a vertical fashion). Initially, the axis of rotation must be centered under the electron beam and positioned at the beams' coincidence point. Locating the axis of rotation on the sample can be achieved by initiating continuous rotation while using the SEM's frame integration option to generate circular 'streaks' in the SEM image. The axis of rotation is at the center of the circular streak marks (s. Figure 2, below). This location can be centered using the microscope's sample stage - e.g. by using the microscope software's 'centering' function. 
The piezo-actuated rotation drive's bearings provide minimal eccentricity during sample rotation. The sample's region of interest is centered on the axis of rotation by applying a linear shift in one dimension, followed by sample rotation by $90^{\circ}$ and another linear shift. The process is repeated at increasingly higher magnifications until the sample is centered on the axis of rotation.

Once the sample is positioned as described above using the 'top-down' SEM view, continuous rotation is initiated while the operator switches to the microscope's FIB view. Then, the specimen under investigation can be sharpened by using a small rectangular milling pattern. The pattern box can be sized and angled in a multitude of configurations in order to generate tips with well-defined shapes and shank angles (such as for APT), or pillars/posts - with parallel edges - for nanomechanical compression testing or X-ray tomography applications.

The images shown in Figure 3 provide an example for sharpening a $\mathrm{Cu}$ post into a tip shape.

\section{References:}

[1] Martin Dierolf, Andreas Menzel, Pierre Thibault, Philipp Schneider, Cameron M. Kewish, Roger Wepf, Oliver Bunk \& Franz Pfeiffer Nature 467, 436-439 (23 September 2010)

doi:10.1038/nature09419
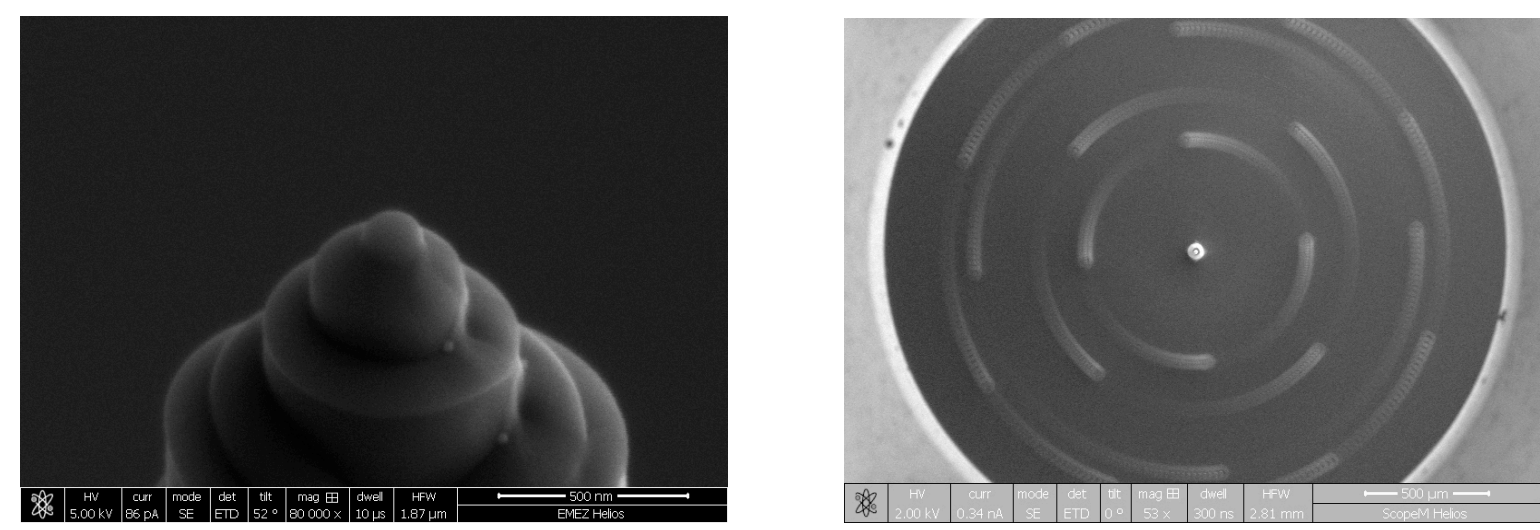

Figure 1 (left): Example of a structure milled with a poorly defined shank angle. Figure 2 (right): Axis of rotation centered in SEM frame of view. Using a long integration time yields streaking which in turn allows easy location of the axis of rotation. One post of an array is centered.
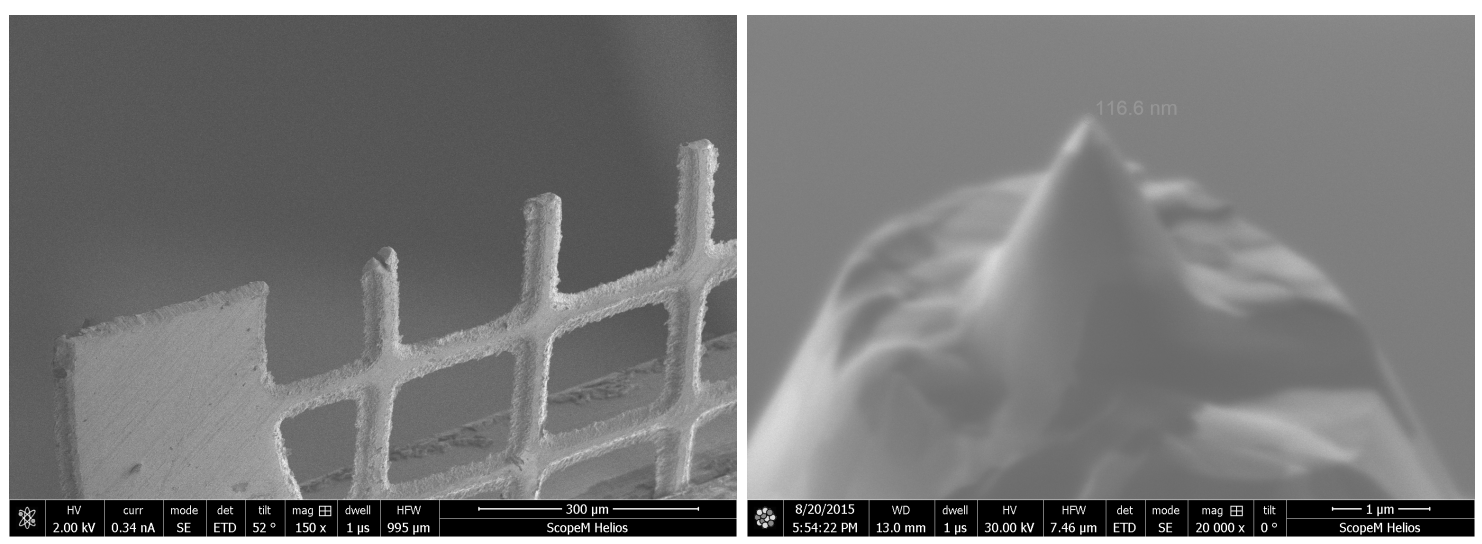

Figure 3: $\mathrm{Cu}$ post after milling using concentric rotation and a rectangular milling pattern. Note the final tip radius is $<60 \mathrm{~nm}$, purely based on mechanical rotation. 